\title{
Introduction to Artificial Intelligence for Sustainable Value Creation
}

\section{Margherita Pagani and Renaud Champion}

"How can artificial intelligence create value in a sustainable way for my business?" is nowadays more than ever the hot topic that every responsible manager needs to cope with to make sure his/her company stays competitive in a highly technology-driven world. For several years there had been many predictions about how artificial intelligence (AI) would change the way we work and how it would have a profound effect on every area of business. However, most of these speculations are not relying on empirical studies or real-life practice. From a business standpoint, AI is still seen as a very young technology with not enough return on experience. But while most companies are still working on their digital transformation, they all start to realize that their "AI transformation" is no longer a futuristic concept but the next challenge they will have to face.

To address this issue, in 2018 at emlyon business school (France) we launched the Institute for Artificial Intelligence in Management, and inside this framework the first Research Center on Artificial Intelligence in Value Creation. Our ambition was to understand the opportunities and implications of AI for the management of organizations, industries, and business ecosystems. To do so, we developed a hybrid approach mixing the rigor of academic research with the pragmatic reality of businesses. Our starting point was to properly define what $\mathrm{AI}$ is referring to. As we will see later on, the recent technological developments make this attempt quite challenging.

Then we decided to consider value creation from a holistic point of view. This implies looking at the impact of AI not only from the economic point of view but also analyzing how it would change the way companies work, are governed, change their business models and reshape their ecosystem. This unique approach led us to consider how this technology can also generate value at the ethical and societal levels. The third key hypothesis was to root our research effort in real-life use cases coming from a broad range of business and management subjects. Only initiating collaborative research that addresses real-world issues facing organizations would allow us to produce a rich seam 
of knowledge and applied solutions relevant to help business leaders navigate through these turbulent times.

This multidisciplinary initiative allowed us to meet and debate with recognized scholars from all over the world in various fields such as Management, Marketing, Information Systems, Data Science, Ethics, and Design. These experts were invited to share their research and open the debate on how AI creates new value layers affecting user behavior and businesses. One of the outputs of the AIM Research Center on AI for Value Creation was the setup of a presentation series to stimulate interdisciplinary research, academicbusiness interactions, and networking. Given the high level of content presented we found it valuable to summarize it in an edited book as a useful tool to disseminate the knowledge and the discussion opened during the presentations with managers, scholars, and the new generation of students. Each of the contributing authors is an authoritative scholar in Strategy, Information Systems, Management, Marketing, Design, or Data Science. We wish to thank all of them for having agreed to share their vision and to contribute to the reflection around Artificial Intelligence for Sustainable Value Creation.

\section{TOWARDS A VALUE-CENTERED APPROACH OF ARTIFICIAL INTELLIGENCE}

During a summer project in 1956 two young mathematicians, John McCarthy from Dartmouth College and Marvin Minsky from MIT, introduced the concept of AI as a research discipline, "to proceed on the basis of the conjecture that every aspect of learning or any other feature of intelligence can in principle be so precisely described that a machine can be made to simulate it." (McCarthy et al. 2006). ${ }^{1}$

For the two young researchers, AI aims at better understanding intelligence, without defining it per se, but breaking it into several cognitive functions. These functions could then be simulated by a computer and used for various practical cases (Ganascia 2017). ${ }^{2}$ Inspired by this early definition, many scientists have been designing algorithms and building devices to mimic humans' senses. They have invented technologies that give machines the capabilities to see, to hear, to speak, to reason, to learn, and to act. Over the years, these prolific developments have led to a vast terminology where the performance of expert systems is compared to machine learning; where weak AI and strong $\mathrm{AI}^{3}$ are debated; where the boundaries between AI, big data, robotics, and the Internet of Things (IoT) ${ }^{4}$ blur. Because of this rich history, it is today quite a challenge to understand what AI has become and what it covers. After 65 years of innovation and scientific discoveries, it is time to clarify what AI is and what managerial and societal value it may bring to companies and individuals. This is necessary, not only to avoid misunderstandings, but also to 
achieve a common knowledge of AI that can be understood by non-AI experts. To this purpose, the multidisciplinary High-Level Expert Group on Artificial Intelligence appointed by the European Commission has proposed the following consensual definition ${ }^{5}$ :

Artificial intelligence (AI) systems are software (and possibly also hardware) designed by humans that, given a complex goal, act in the physical or digital dimension by perceiving their environment through data acquisition, interpreting the collected structured or unstructured data, reasoning on the knowledge, or processing the information, derived from this data and deciding the best action(s) to take to achieve the given goal. AI systems can either use symbolic rules or learn a numeric model, and they can also adapt their behavior by analyzing how the environment is affected by their previous actions.

However, to reveal the full potential of AI, we believe the purely technical approach is not enough. For sure, AI can transform the world for the better. It can improve healthcare from diagnosis to surgery and rehabilitation (e.g., AI-empowered radiology, robotic-assisted minimally invasive surgery, intelligent prosthesis). It can help produce goods in a sustainable way with smart manufacturing tools relying on an optimized supply-chain (e.g., Radio Frequency Identification, ${ }^{6}$ tagging of goods, automated forklifts and Automated Guided Vehicles ${ }^{7}$ in warehouses, lightweight robotics arms on production lines). It can personalize our education according to our lifelong learning needs (e.g., digital personal assistants, on-demand learning platforms, blockchain certification of diplomas). It can also redesign our cities to make them safer and more energy-efficient (e.g., autonomous vehicles, connected infrastructure based on Building Information Modeling (BIM) ${ }^{8}$, face recognition and biometric sensors for threat detection). But while AI can positively impact the whole society and the economy, it is also raising many ethical questions.

- Will AI generate massive unemployment due to generalized automation?

- Will it threaten our democracies by creating a society of permanent and invasive control?

- Will it segment humanity by augmenting a selected few instead of repairing the many who need it? ...

We believe that these threats will be overcome only if human wellbeing and the common good are put at the center. To do so, AI systems need to follow a value-centered approach, with the ultimate goal of improving human welfare and freedom. All stakeholders from engineers to consumers and policymakers need to ensure that human values and societal issues are central to how such systems are developed, used and monitored. This is the reason why we call for a human-centric AI which adds an ethical dimension to its technical features. 


\section{THE VALUE CREATION FRAMEWORK}

The impact of new technology is measured most of the time in terms of the economic value it generates, which is translated into efficiencies and effectiveness in the business processes. This is the case for AI systems that allow internal and external business operations to be optimized, better insights from data analysis to be gained, or the features, functions of products to be enhanced, also allowing new markets to be pursued. However, in the approach of AI we promote, this is just one dimension of value creation. When AI systems interact and collaborate with people to enhance human abilities and empower both individuals and society, the value is created at three different levels: ethical, business and societal:

- ethical value, by guaranteeing social fairness in respect of human freedoms and dignity, by fostering explicability, or by ensuring prevention of harm;

- business value, by becoming part of the products themselves, by improving performance across the value chain, or by enabling ecosystems and collaboration with new markets;

- societal value, by including environmental sustainability, by encouraging a new social contract based on accountability, or by building a safer world.

In the following chapters, we explore not only how AI systems create value inside organizations alongside these main value components, but we also analyze how a virtuous circle of value creation can be initiated. Indeed each level of value creation can induce changes and opportunities at the consecutive levels. For instance, ethical concerns can influence new business models and enforce new business practices. Businesses produce innovations that have an impact on the whole society and the environment, including new products and services, new jobs and work organization, as well as new energy-efficient production processes. These societal changes can consequently induce risks and opportunities that should be addressed ethically. These dynamics are at the center of the framework we propose to adopt when looking at AI systems (Figure 0.1).

The book is divided into seven chapters organized around three main parts: I. Human-Centric AI; II. Business Value; III. Ethical and Societal Value.

In Part I we lay the foundations defining human-centric AI as a pervasive economic, societal, and organizational phenomenon.

Chapter 1, "Creating business value through human-centric AI", first defines human-centric AI and classifies AI systems into four distinct categories. Each presents its own set of challenges and required skillsets to enable effective collaboration between humans and machines. Examples of AI systems include robotics and autonomous vehicles, facial recognition, natural language 


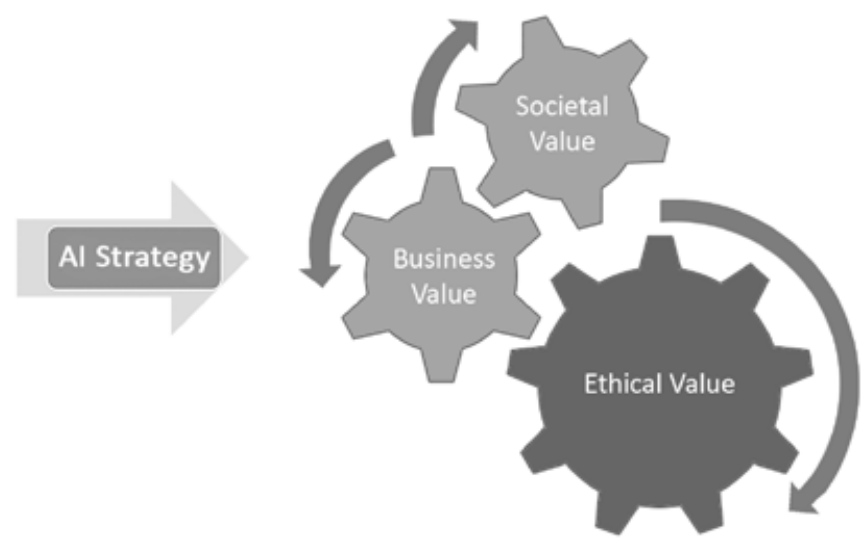

Figure $0.1 \quad$ The value creation framework

processing, virtual agents, and machine learning, which are being deployed in a variety of problem domains ranging from cybersecurity to fintech and from education to healthcare. These different AI systems may bring value in different ways and may require different shared responsibilities with humans.

Chapter 2, "Value-driven design of AI enabled experiences", focuses on how AI is reshaping the way we experience the tangible world. The chapter illustrates real case examples from work done at MIT Design Lab, with a focus on design methodologies adopted at the Lab to envision meaningful experiences between people and intelligent systems.

In Part II we focus more on the business value and the unprecedented opportunities provided by AI for designing intelligent products, devising novel service offerings, and inventing new business models and organizational forms.

Chapter 3, "Digital platform ecosystems: the coming context for AI", examines the role of AI in platform ecosystems, highlighting both how AI might be used to increase the value created by these platforms, but also the challenges that arise as AI becomes central to the governance of these AI ecosystems.

Chapter 4, "Unlocking value from AI in financial services: strategic and organizational tradeoffs vs. media narratives" develops a general conceptual model of the strategic and organizational tradeoffs inherent in extracting value from AI.

Accompanying the horizon of possibilities, in Part III the emerging and complex challenges around privacy, security and ethics are explored.

Chapter 5, "The challenge of responsible AI", presents the concept of ethics and its application to AI systems and describes different initiatives to tackle 
the issue of negative consequences of AI, providing guidelines for managers to address the issue of ethics in AI, including metrics for corporate social responsibility.

Chapter 6, "A model of fair and explainable artificial intelligence", addresses fairness in the algorithm presenting a novel theory-based individual-level dynamic learning method that performs learning using data of an individual subject without employing others' information and identifies a causal mechanism from the unobserved data generating process that each subject exhibits.

In Chapter 7, "Ethical maintenance of artificial intelligence systems" introduces Z-Inspection $\AA$, a reference and a process model for AI ethical maintenance, to help monitor AI systems after deployment, preserve ethical principles, and ensure robustness, from both a technical and social perspective, within the context of given legal and contractual frameworks.

Finally, the Afterword opens up new research avenues discussing the value of AI putting the human at the center.

\section{THE PROMISE OF THIS BOOK}

Whereas the transformative potential of AI is widely recognized, there is significant uncertainty for businesses on how to manage AI and its implications. AI raises new questions and creates new opportunities and it is more and more advantageous to carefully consider empirical and theoretical work. On a theoretical front, $\mathrm{AI}$ is a domain rife with opportunities for generating novel perspectives for managing information systems, marketing, strategy, and new business models, together with novel theorizing about the AI artifact. From a methodological perspective, AI can enable us to analyze unprecedented reams of digital data in new and interesting ways - potentially opening up new avenues to theory generation (Lazer et al. 2009) ${ }^{9}-$ while at the same time addressing challenges associated with such novel approaches (Berente et al. 2019; Howison et al. 2011) $)^{10}$.

The book aims to provide in-depth analyses and studies of applications of AI to real-world problems. Each chapter aims to provide an original, critical and accessible account of the current state of the debate in its domain. The uniqueness of this edited book is the focus on value creation, a subject of great importance but still not addressed by existing publications. Moreover, the book, using a multidisciplinary perspective, aims to address the interests of a wide spectrum of practitioners, students, and researchers alike who are interested in identifying the value generated by AI systems in management and discover opportunities and challenges. 


\section{NOTES}

1. McCarthy, J., Minsky, M.L., Rochester, N., and Shannon, C.E. (2006). 'A Proposal for the Dartmouth Summer Research Project on Artificial Intelligence.' August 31, 1955. AI Magazine, 27(4), 12.

2. Ganascia, J.G., (2017). Le Mythe de la Singularité, Paris, France: Seuil.

3. Weak $A I$ is a term used to describe artificial intelligence using sensors and algorithms to simulate a limited set of cognitive functions to solve a specific problem. Strong $A I$ refers to the capacity of artificial intelligence to apply intelligence to any problem sometimes involving consciousness.

4. The Internet of Things stands for objects with computing devices in them that are able to connect to each other and exchange data using the internet (source: Cambridge Dictionary).

5. High-Level Expert Group on Artificial Intelligence, (2019). A Definition of AI: Main Capabilities and Scientific Disciplines, Brussels: European Commission.

6. Radio Frequency Identification is used as a small electronic device fixed to goods sending a radio signal with information that can be processed by a computer.

7. Automated Guided Vehicles are mobile robots using sensors to navigate.

8. Building Information Modeling is an intelligent 3D model-based process that gives architecture, engineering and construction professionals the insight and tools to plan, design, construct and manage buildings and infrastructure more efficiently (source: Autodesk).

9. Lazer, D., Pentland, A., Adamic, L., Aral, S., Barabási, A-L, Brewer, D., Christakis, N. et al. (2009). Computational social science. Science, 323(5915):721-3.

10. Berente, N., Seidel, S. and Safadi, H. (2019). Data-driven computationally intensive theory development. Information Systems Research, 30(1), 50-64; Howison, J., Wiggins, A. and Crowston, K. (2011). Validity issues in the use of social network analysis with digital trace data. Journal of the Association for Information Systems, 12(12), 1-15. 